\title{
Single device transcatheter closure for double atrial septal defect under real time three-dimensional image guidance
}

Young Kyoung $\mathrm{Sa}^{1}$, Chul Soo Park ${ }^{1}$, Eun Joo $\mathrm{Cho}^{1}$, Yoon Seok $\mathrm{Choi}^{2}$, and Heung Seok Kim

${ }^{1}$ Division of Cardiology, Department of Internal Medicine, Yeouido St. Mary's Hospital, College of Medicine, The Catholic University of Korea, Seoul; ${ }^{2}$ Division of Cardiology, Department of Internal Medicine, Seoul St. Mary's Hospital, College of Medicine, The Catholic University of Korea, Seoul, Korea

Received: February 16, 2021 Revised : February 26, 2021 Accepted: March 22, 2021

\section{Correspondence to} Chul Soo Park, M.D.

Tel: +82-2-3779-1447

Fax: $+82-2-783-8516$

E-mail: charlie@catholic.ac.kr https://orcid.org/0000-00033048-3288
A 6o-year-old male patient was admitted for exertional dyspnea. Transthoracic and transesophageal echocardiogram (TEE) revealed secundum atrial septal defect (ASD) (Fig. 1). The defect was oval shaped and about $10 \mathrm{~mm}$ in the maximum diameter. However, shunt flow was observed as dual jet by Color Doppler at a certain degree view by TEE, suggesting multi-hole morphology, while transthoracic echocardiogram and computed tomography did not suggest any complexity of the defect. Double fenestrations of the interatrial septum (IAS) were clearly observed by real time three-dimensional (3D) TEE (Fig. 2 and Supplementary Videos 1 and 2). The deformity had two holes, one of which was round, close to the aortic rim, and in a relatively low position, while the other was crescent shaped and in an upper position. Each defect was about $5 \mathrm{~mm}$ in size.
Multi-fenestration is a well-known hurdle to transcatheter ASD closure. A small additional defect adjacent to the larger one can also be closed by single device implantation through the major defect. However, in this case, it was difficult to select a closing strategy because single instrument does not guarantee all-encompassing closure for both sizable defects.

The heart team chose a $12 \mathrm{~mm}$-diameter Amplatzer Septal Occluder (Abbott, St. Paul, MN, USA) and determined cannulation to the round hole, considering which one was mechanically durable and anatomically fit to the device neck. Wire crossing to the round hole was performed successfully under real time $3 \mathrm{D}$ TEE guidance. Thereafter the occluder was deployed uneventfully (Supplementary Videos 3 and 4). Shunt flow was resolved without structural deterioration of IAS af-
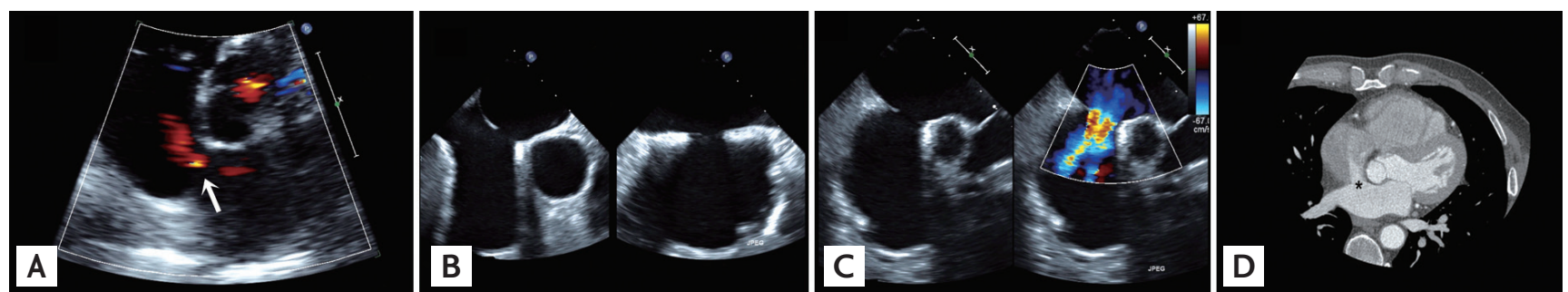

Figure 1. Interatrial septal defect and shunt flow (arrow) suspected by transthoracic echocardiography (A). Confirmed defect (B) and double jet shunt flow (C) shown by transesophageal echocardiography. Contrast leakage (asterisk) from left atrium to right atrium documented by computed tomography (D). 

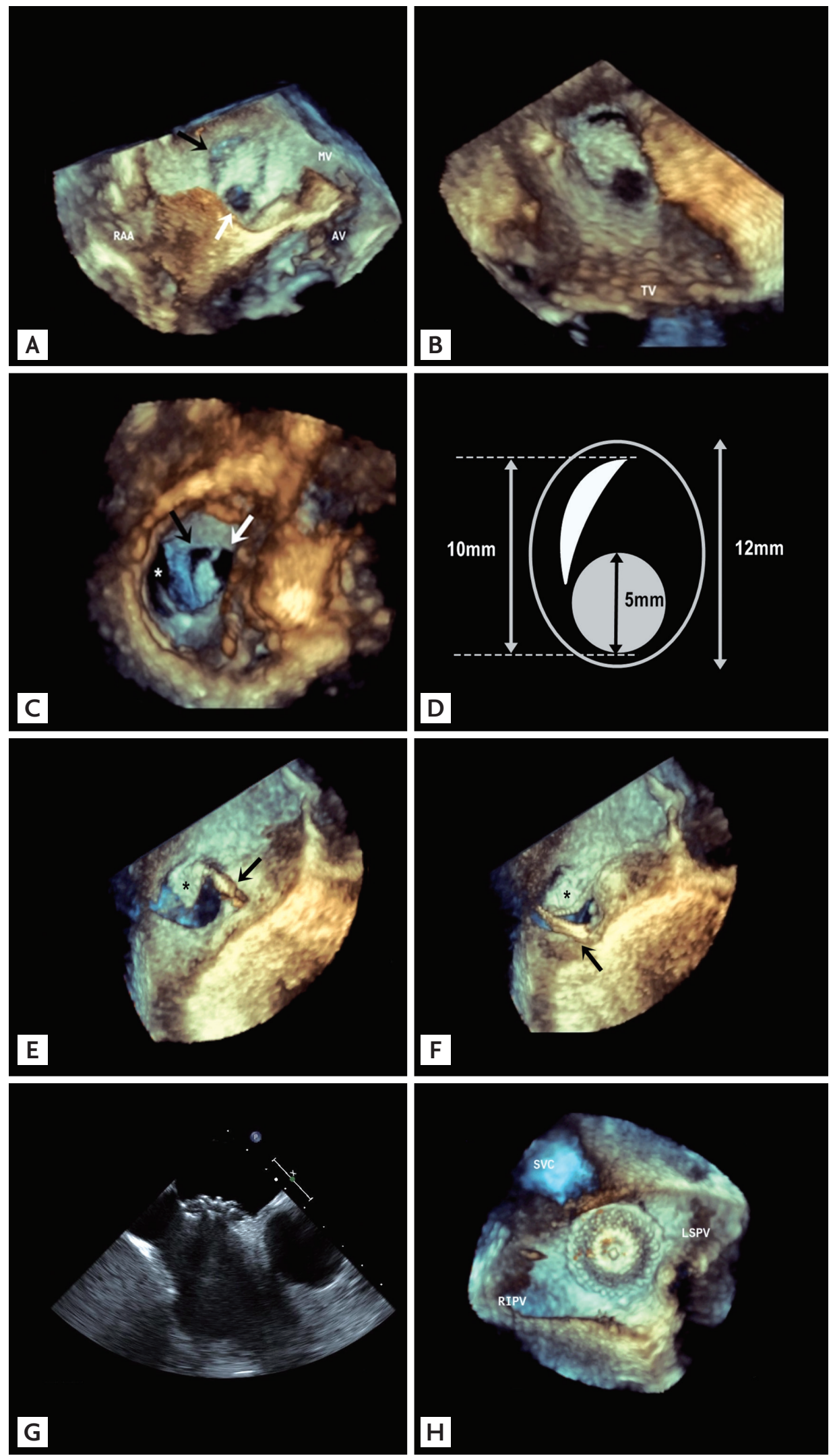

Figure 2. Real time three-dimensional transesophageal echocardiography showing round (white arrow) and crescent-shaped (black arrow) defects in the fossa ovalis viewed from left atrium (A), right atrium (B), and inferior vena cava (C; asterisk, superior vena cava). Diagrammatic illustration with sizing (D). The tapered end of the intervening strand (asterisks) between two apertures flexibly tented by crossing-wire (arrows) through the round hole according to cardiac cycles (E, F). The implanted device $(\mathrm{G}, \mathrm{H})$. RAA, right atrial appendage; $\mathrm{MV}$, mitral valve; $\mathrm{AV}$, aortic valve; TV, tricuspid valve; SVC, superior vena cava; LSPV, left superior pulmonary vein; RIPV, right inferior pulmonary vein. 
ter device implantation.

This case illustrated unique ASD images viewed from various positions. $3 \mathrm{D}$ TEE is a highly informative and superior image modality for display anatomy of complex ASD and real time guidance of transcatheter closure. In conclusion, similar sized-double ASD can be treated with a single device under real time ${ }_{3} \mathrm{D}$ TEE guidance in selected cases.

\section{Conflict of interest}

No potential conflict of interest relevant to this article was reported. 
Oh S, et al. Gigantic thoracic aortic aneurysm

Supplementary Video 1. Double atrial septal defect (ASD) comprised of round and crescent shaped defects. SVC, superior vena cave; IAS, interatrial septum; AV, aortic valve.

Supplementary Video 2. Atrial septal defect (ASD) looked up from inferior vena cava (IVC). SVC, superior vena cave.

Supplementary Video 3. Guidewire inserted through round pivotal defect.

Supplementary Video 4. Implanted device. SVC, superior vena cava; RIPV, right inferior pulmonary vein; LSPV, left superior pulmonary vein. 